\title{
Complete metastasectomy in renal cell carcinoma: a propensity-score matched by the International Metastatic RCC Database Consortium prognostic model
}

\author{
Aline F Fares ${ }^{1,2,}$, , Daniel V Araujo ${ }^{1,2,}$, , Vinicius Calsavara ${ }^{3}$, Augusto Obuti Saito ${ }^{1}$, Maria Nirvana Formiga ${ }^{1}$, Aldo A Dettino ${ }^{1}$, Stenio Zequi ${ }^{4}$, \\ Walter $\mathrm{H}$ da Costa ${ }^{4,5}$ and Isabela W Cunha ${ }^{6,7}$
}

${ }^{1}$ Department of Medical Oncology, AC Camargo Cancer Center, São Paulo 01525-001, Brazil ${ }^{2}$ Department of Medical Oncology, University Health Network, Toronto, ON M5G 1L7, Canada

${ }^{3}$ Department of Epidemiology and Statistics, AC Camargo Cancer Center, São Paulo 01525-001, Brazil

${ }^{4}$ Department of Urology, AC Camargo Cancer Center, São Paulo 01525-001, Brazil

${ }^{5}$ Department of Urology, Santa Casa de Misericordia de São Paulo, São Paulo 01525-001, Brazil

${ }^{6}$ Department of Pathology, AC Camargo Cancer Center, São Paulo 01525-001, Brazil

${ }^{7}$ Department of Pathology, Rede D'OR-Sao Luis, São Paulo 01525-001, Brazil

*Aline F Fares and Daniel $\mathrm{V}$ Araujo contributed equally to writing this article.

This study was performed at the AC Camargo Cancer Center.

\section{Abstract}

Introduction: We evaluated overall survival (OS) benefit of complete metastasectomy (CM) in metastatic renal cell carcinoma ( $\mathrm{mRCC}$ ) using a propensity score-matched (PSM) analysis to balance groups by age, gender and by the International Metastatic RCC Database Consortium prognostic model (IMDC).

Methods: We included patients (pts) treated at the AC Camargo Cancer Center between 2007 and 2016. Pairs were matched by age, gender and IMDC. Kaplan-Meier survival estimates and Cox proportional hazard models were used to evaluate OS on CM and no-CM group.

Results: We found 116 pts with clear cell mRCC. After PSM, the number was reduced to 74 (37 CM, 37 no-CM). The median OS for CM and no-CM was 98.3 months and 40.5 months, respectively (hazard ratio $0.2495 \% \mathrm{Cl} 0.11-0.53 p<0.001$ ). The OS benefit of CM was confirmed on favourable and intermediate IMDC but was absent on poor IMDC. The CM group received less systemic therapy than the no-CM group. Ten pts in the CM group still have no evidence of disease (NED).

Conclusion: After matching for age, gender and IMDC, we found CM impacts on OS and also diminishes the need for systemic treatment. Survival benefit was confirmed for favourable/intermediate IMDC but not for the poor IMDC prognostic model. Further studies correlating IMDC and metastasectomy are needed to guide clinical decision-making.

Keywords: metastasectomy, clear cell renal cell carcinoma, targeted therapy, propensity score matching

Correspondence to: Dr Aline F Fares Email: aline.fares@uhn.ca

ecancer 2019, 13:967

https://doi.org/10.3332/ecancer.2019.967

Published: $14 / 10 / 2019$

Received: 12/05/2019

Publication costs for this article were supported by ecancer (UK Charity number 1176307).

Copyright: (C) the authors; licensee ecancermedicalscience. This is an Open Access article distributed under the terms of the Creative Commons Attribution License (http:// creativecommons.org/licenses/by/3.0), which permits unrestricted use, distribution, and reproduction in any medium, provided the original work is properly cited. 


\section{Introduction}

The treatment of metastatic renal cell carcinoma (mRCC) has changed dramatically over the last 30 years. Following an evolution from interleukin and interferon to tyrosine kinase inhibitors (TKI) and immunotherapy, survival rates have risen significantly [1]. Nevertheless, cure with systemic treatments alone is elusive for the vast majority of patients, and local treatments remain an important treatment pillar often pursued whenever possible.

Oligometastatic RCC can be treated radically as a localised disease [2]. Surgery or stereotactic radiosurgery of metastatic sites can be offered aiming to prolong time-to-treatment and OS [2, 3]. Usually, these treatments are offered to a selected population with good performance status and fit enough to undergo surgical interventions with an underlying active cancer. Prior reports, mostly retrospective, found improvements in survival, showing 5-year survival rates of up to $80 \%$ in patients undergoing metastasectomy, with variations depending on site of metastasectomy [4-9]. Lung and pancreatic metastasectomies appear to have higher rates of success and better survival, whereas bone and liver are related to worse survival $[10,11]$.

Although these studies report impressive survival numbers, the question of whether metastasectomy per se is directly associated with survival or if disease biology of these indolent and slow progressor tumours is the main factor, is yet to be answered. Verbiest et al [12] evaluated if the molecular subtypes ccRCC1-4 are prognostic in the setting of CM. They studied 43 clear cell RCC (ccRCC) patients and found subtype-dependent impact in terms of disease relapse after CM: ccRCC1\&4 have 5-year disease-free survival (DFS) of 10\%, whereas ccRCC2\&3 have 5-year DFS of $27 \%$. Certainly, this supports the importance of tumour biology underlying metastasectomy benefit and suggests that ccRCC1\&4 might be better treated with systemic treatment alone. However, these subtypes are obtained by RNA-sequencing, a resource not readily available, and that can bring financial challenges to clinical practice.

A promptly available method to evaluate prognostic in mRCC is the International Metastatic Renal Cell Carcinoma Consortium (IMDC) risk factor [13]. It includes clinicolaboratorial data that categorise patients in favourable/intermediate/poor prognosis and is widely used in clinical trials and clinical practice to advise therapeutical guidelines. Cytoreductive nephrectomy (CN) was evaluated in a large cohort of synchronous metastatic patients and was found to impact on better survival for patients with 0-3 IMDC criteria; those with 4-6 IMDC criteria do not derive survival benefit from CN [14]. IMDC is also used for treatment decision-making in first-line systemic treatment for mRCC and as of today, firstline treatment as per guidelines is nivolumab and ipilimumab for intermediate/poor prognosis and TKI for favourable prognosis [15, 16].

Despite the clear importance of IMDC criteria as a clinical tool for decision-making, to the best of our knowledge, there are no reports evaluating the role of metastasectomy stratified per IMDC prognostic groups. In this study, we investigate the role of metastasectomy in a PSM population accounting for known prognostic factors in mRCC such as age, gender and IMDC criteria.

\section{Materials and methods}

The present study was conducted after approval of the AC Camargo Cancer Center institutional ethics committee. Using the ACC biobank, we searched for metastatic tissue samples in RCC. We included patients with clear cell histology and absence of sarcomatoid or rhabdoid features who underwent previous nephrectomy. During radical nephrectomy, retroperitoneal lymphadenectomy was restricted to the renal hilum and was performed for staging purposes only. In nephron-sparing procedures, lymph node dissection was not performed. We included patients who underwent $\mathrm{CM}$ and also patients treated without metastasectomy and with targeted therapy (TT) alone who fit inclusion criteria.

Complete metastasectomy (CM) was defined as no evidence of residual disease following surgery, neither on index organ nor other organs and therefore considered a curative intent surgery. The decision to perform CM was at the discretion of the treating physician and patient acceptance. CM was generally advised for patients with limited resectable disease and low cardiovascular surgery risk. The surgery could have been done at any time during the treatment course. Metastases locations sites were classified as: lungs, bones, liver, central nervous system (CNS), lymph nodes, intra-abdominal (including pancreas, adrenal, abdominal lymph nodes) and skin and subcutaneous tissue.

Clinicopathological collected information were: age, gender, Fuhrman/International Society of Urological Pathology (ISUP) grade, site of CM, date of first diagnosis, date of recurrence, status and date of the last follow-up. Karnofsky performance scale (KPS), calcium, neutrophils and platelets values, as well as time from the first diagnosis to metastasis, were annotated as independent variables and as part of the IMDC 
risk criteria. Baseline blood analysis was collected at the time of metastases diagnosis. Patients were then categorised according to IMDC prognosis criteria into good, intermediate or poor prognosis [18]. Staging was assigned using the 2010 American Joint Committee on Cancer (AJCC) classification.

We defined synchronous metastasis as patients who had distant metastasis diagnosed upfront or within 6 months from the first diagnosis. Recurrence was defined as a new site of a metastatic disease diagnosed clinically, by imaging or biopsy.

All patients underwent a metastatic workup before metastasectomy, to rule out other sites of metastases. This included CT of the chest, abdomen and pelvis in all patients; bone scan and brain MRI if clinically advised. Systemic treatment after metastasectomy could be administered, at the discretion of the treating physician. Patient information was obtained from the AC Camargo's electronic medical chart. Surveillance after CM typically consisted of CT scan of the chest, abdomen and pelvis every 4 months for the first 2 years and every 6 months consequently, as per institutional policy.

\section{Statistical analysis}

We matched and balanced CM/no-CM in pairs for age, gender and IMDC risk factor. Matching and balancing of the empirical distributions were done using the Matchlt package in R, version 3.1.1 (https://www.r-project.org/). This propensity score analysis helps to make CM/no-CM homogeneous in terms of age, gender and IMDC risk factor. Hence, it provides less bias in the evaluation of metastasectomy impact on OS.

Association between clinicopathological variables and CM status were performed by chi-square and Fisher's exact test, as adequate. KaplanMeier curves were used to predict OS and the curves were compared using log-rank test. Cox proportional hazards regression model was used to assess the prognostic significance of CM in univariable and multivariable analysis, adjusting for CNS metastasis, bone metastasis, intra-abdominal metastasis and IMDC criteria. These variables were selected based on statistical significance in the univariable model. $p$ values are derived from two-tailed tests. All analyses were performed using SPSS v24 and software R version 3.5.

\section{Results}

\section{Patients demographics}

Of all patients diagnosed with mRCC between 2007 and 2016, 116 matched study inclusion criteria. 70\% were male and the median age was 57 years (32-82). Synchronous metastasis was present in 41 patients (35\%).

After matching pairs for age, gender and IMDC criteria, groups were reduced to 74 patients: 37 on the CM group and 37 on the no-CM group as depicted in the flowchart (Figure 1). From the cohort of paired patients, 21 patients (28\%) had synchronous metastasis; 53 (72\%) patients had localised disease at the time of diagnosis and developed further metastases 6 months or more after the first diagnosis of RCC.

After the matched paired analysis, clinical and prognostic characteristics were well balanced between CM and no-CM groups as shown in Table 1. There was no association between IMDC criteria and CM ( $p=1.0)$. Twenty-one patients (28\%) were classified as IMDC good prognosis, 49 (66\%) were intermediate and $4(5 \%)$ were poor prognosis. The most common site of metastasis was lung ( $86 \%)$, followed by bones (50\%), lymph nodes (39\%), intra-abdominal metastasis (30\%), liver (22\%), CNS (22\%) and skin and subcutaneous (18\%), as shown in Table 2. The most common sites of metastasectomy were lungs (84\%), bones (49\%), intra-abdominal (32\%), lymph nodes (30\%), liver (27\%), skin and subcutaneous (16\%) and CNS (13\%). From all 34 patients in the CM group, 20 (59\%) underwent one session of metastasectomy, 11 (32\%) received two sessions and 6 (18\%) received three or four sessions of metastasectomy. CNS was the only site of metastasis where we found inverse correlation with metastasectomy, likely because patients with CNS metastasis are usually not in a good performance to undergo metastasectomy or because it is a technically challenging site to perform CM (Table 2). Others sites of metastasis had no correlation with metastasectomy.

From all 74 patients, 62 patients (84\%) received TT at some point of their disease course. Of note, all 12 (16\%) who never received systemic therapy were in the CM group: 4 (33\%) never recurred and have NED; 5 (42\%) had recurrence and underwent a second metastasectomy and 
are now on NED status; 1 (8\%) underwent two additional metastasectomies and is now on NED status as well; the other 2 (16\%) recurred and did not receive further treatment due to low KPS or patient preference.

With respect to the type of systemic therapy, 43 patients (69\%) received sunitinib as first-line therapy, 17 patients (27\%) received pazopanib and 3 (5\%) received temsirolimus. Table 3 summarises types of TT for systemic treatment per IMDC criteria.

Of note, from the full cohort of 74, only 40 (54\%) initiated the second line of systemic therapy and from these, 28 (70\%) were in the No-CM cohort, whereas only 12 (30\%) of the CM cohort received the second line of systemic treatment. 12 patients (30\%) from the CM group and 28 patients (70\%) from the no-CM group, as shown in Table 4. Of these, 14 patients (35\%) received everolimus, 7 patients (17.5\%) received pazopanib and the others received other TT, immunotherapy or even chemotherapy.

\section{Survival outcomes}

After a median follow-up of 70.4 months, median OS for the paired-up population was 78 months. At the time of analysis, 35 patients (46.7\%) had died: 16 patients (47\%) from the CM group and 19 (56\%) patients from the no-CM group.

Comparing the CM group with the no-CM group, median OS was 98.3 months versus 40.5 months (hazard ratio (HR) $0.2495 \% \mathrm{Cl} 0.11-0.53 p$ $<0.0001$ ) respectively, as shown in Figure 2. When the cohort is segregated into IMDC risk groups, there was a significant survival difference between favourable prognosis versus intermediate versus poor prognosis ( 88.05 months, 59.72 months and 18.03 months, respectively; HR $0.1595 \% \mathrm{Cl} 0.04-0.52 p=0.002$ ). There were no significant statistical differences between synchronous or metachronous metastases, probably because groups were paired according to time from diagnosis to treatment less than 1 year, one of the IMDC criteria $(\mathrm{HR} 1.0195 \% \mathrm{Cl}$ $0.69-1.46 p=0.96$ ).

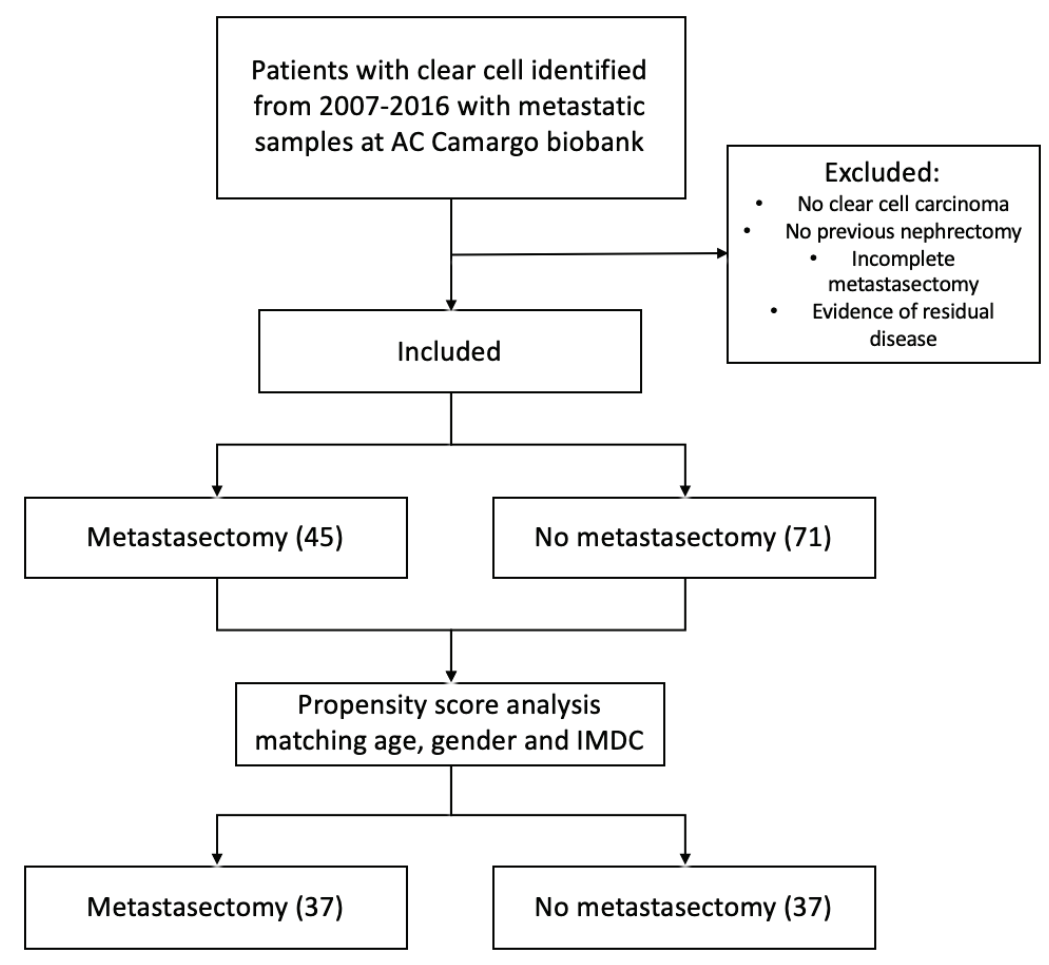

Figure 1. Exclusion process flow chart and propensity score analysis. 
Table 1. Patients' characteristics by $\mathrm{CM}$ versus no-CM.

\begin{tabular}{|c|c|c|c|c|}
\hline Characteristics & Category & $\mathrm{CM}(n=37)$ & No-CM $(n=37)$ & $p$ \\
\hline Age & Mean (SD) & $55.92(11 \%)$ & $57.38(11 \%)$ & 0.575 \\
\hline \multirow[t]{2}{*}{ Sex } & Female & $12(32 \%)$ & $9(24 \%)$ & \multirow[t]{2}{*}{0.606} \\
\hline & Male & $25(68 \%)$ & $28(76 \%)$ & \\
\hline \multirow[t]{4}{*}{ Fhurman } & 1 & $2(8 \%)$ & $2(8 \%)$ & \multirow[t]{4}{*}{0.879} \\
\hline & 2 & $10(42 \%)$ & $10(42 \%)$ & \\
\hline & 3 & $5(21 \%)$ & $7(29 \%)$ & \\
\hline & 4 & $7(29 \%)$ & $5(21 \%)$ & \\
\hline \multirow[t]{3}{*}{ IMDC criteria } & Good & $11(30 \%)$ & $10(27 \%)$ & \multirow[t]{3}{*}{1} \\
\hline & Intermediate & $24(65 \%)$ & $25(68 \%)$ & \\
\hline & Poor & $2(5 \%)$ & $2(5 \%)$ & \\
\hline Metastasis status & $\begin{array}{l}\text { Synchronous } \\
\text { Metachronous }\end{array}$ & $\begin{array}{l}14(37 \%) \\
23(62 \%)\end{array}$ & $\begin{array}{c}7(19 \%) \\
30(81 \%)\end{array}$ & 0.06 \\
\hline Systemic therapy at any time & $\begin{array}{l}\text { No } \\
\text { Yes }\end{array}$ & $\begin{array}{l}12(32 \%) \\
25(68 \%)\end{array}$ & $\begin{array}{c}0(0 \%) \\
37(100 \%)\end{array}$ & $<0.0001$ \\
\hline Number of metastasectomy sessions & $\begin{array}{l}1 \\
2 \\
3 \text { or } 4\end{array}$ & $\begin{array}{c}20(59 \%) \\
11(32 \%) \\
6(18 \%)\end{array}$ & $\begin{array}{l}0 \\
0 \\
0\end{array}$ & \\
\hline
\end{tabular}

Table 2. Metastatic sites and metastasectomy sites.

\begin{tabular}{|l|c|c|c|c|}
\hline Metastasectomy site & All N (\%) & CM N (\%) & No-CM N (\%) & $p$ \\
\hline All sites & $74(100 \%)$ & $37(100 \%)$ & $37(100 \%)$ & \\
\hline Lungs & $64(86 \%)$ & $31(84 \%)$ & $33(89 \%)$ & 0.36 \\
\hline Bones & $34(50 \%)$ & $18(49 \%)$ & $19(51 \%)$ & 0.40 \\
\hline Liver & $16(22 \%)$ & $10(27 \%)$ & $6(16 \%)$ & 0.19 \\
\hline CNS & $16(22 \%)$ & $5(13.5 \%)$ & $11(29 \%)$ & 0.02 \\
\hline Lymph nodes & $29(39 \%)$ & $11(30 \%)$ & $18(48 \%)$ & 0.07 \\
\hline Intra-abdominal & $22(30 \%)$ & $12(32 \%)$ & $10(27 \%)$ & 0.50 \\
\hline Skin & $13(18 \%)$ & $6(16 \%)$ & $7(18 \%)$ & 0.38 \\
\hline
\end{tabular}

On univariable analysis, CNS and bone metastases were associated with worse OS, whereas intra-abdominal metastases were associated

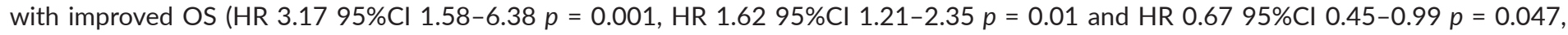
respectively) as depicted in Figure 3A, 3B and 3C. On multivariable analysis, even though IMDC criteria had a trend towards statistical significance ( $\mathrm{HR} 0.2995 \% \mathrm{Cl} 0.07-1.1 p=0.08$ ), CM was the only significant variable ( $\mathrm{HR} 0.2195 \% \mathrm{Cl} 0.09-0.49 p<0.0001)$, after adjusting for IMDC, bone metastasis, CNS metastasis and intra-abdominal metastasis (Table 5).

We also analysed the impact of CM on OS for good/intermediate and for poor IMDC prognosis group separately. The benefit was confirmed for the first group, (HR $0.2295 \% \mathrm{Cl} 0.09-0.5 p<0.0001$ ). However, for the poor prognosis group, there was no benefit added by CM (HR $1.6195 \% \mathrm{Cl} 0.14-18.31 p=0.69)$. 
Table 3. Systemic treatment received by IMDC risk factor.

\begin{tabular}{|l|c|c|c|}
\hline \multicolumn{1}{|c|}{ Type of systemic treatment used in first line } & \multicolumn{3}{c|}{ IMDC risk factor } \\
\hline Sunitinib & Favourable (21) & Intermediate (49) & Poor (4) \\
\hline Pazopanib & $12(57 \%)$ & $27(55 \%)$ & $4(100 \%)$ \\
\hline Temsirolimus & $4(19 \%)$ & $13(27 \%)$ & $0(0 \%)$ \\
\hline No treatment & $1(5 \%)$ & $2(4 \%)$ & $0(0 \%)$ \\
\hline
\end{tabular}

Table 4. Line of systemic therapy received in CM and no-CM groups.

\begin{tabular}{|l|c|c|c|c|}
\hline \multicolumn{1}{|c|}{ Line of systemic treatment } & All N (\%) & CM N (\%) & No-CM N (\%) & $p$ \\
\hline \multicolumn{1}{|c|}{ Alinest line } & $74(100 \%)$ & $37(100 \%)$ & $37(100 \%)$ & \\
Yes & $62(84 \%)$ & $25(68 \%)$ & $37(100 \%)$ & $<0.0001$ \\
No & $12(16 \%)$ & $12(32 \%)$ & $0(0 \%)$ & \\
\hline Second line & & & & $<0.0001$ \\
Yes & $40(54 \%)$ & $12(32 \%)$ & $28(76 \%)$ & \\
No & $34(46 \%)$ & $25(68 \%)$ & $9(24 \%)$ & \\
\hline
\end{tabular}

$\mathrm{CM}+$ Yes + No

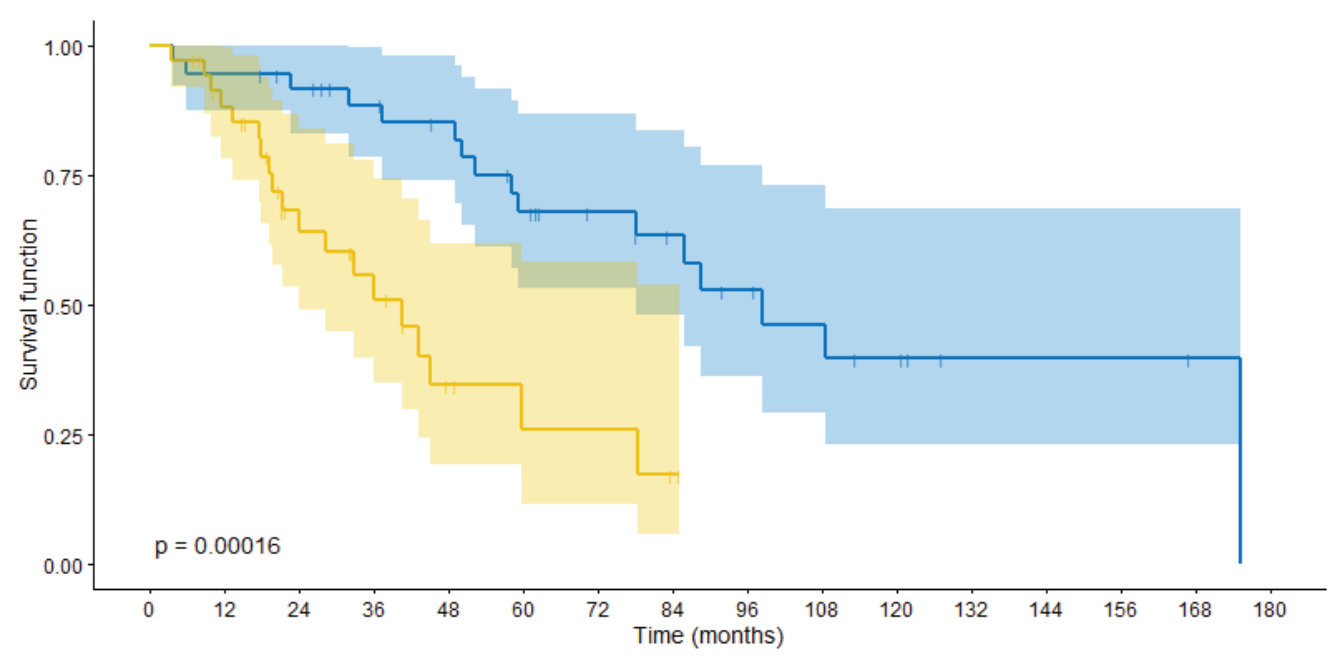

ป Yes- \begin{tabular}{cccccccccccccccc} 
Number at risk \\
37 & 35 & 32 & 28 & 25 & 19 & 15 & 12 & 9 & 7 & 5 & 2 & 2 & 2 & 1 & 0 \\
36 & 29 & 16 & 12 & 5 & 3 & 3 & 1 & 0 & 0 & 0 & 0 & 0 & 0 & 0 & 0 \\
\hline 0 & 12 & 24 & 36 & 48 & 60 & 72 & $\begin{array}{c}84 \\
\text { Time (months) }\end{array}$ & $\begin{array}{c}96 \\
\text { Time }\end{array}$ & 120 & 132 & 144 & 156 & 168 & 180
\end{tabular}

Figure 2. OS comparing CM group with no-CM group. 

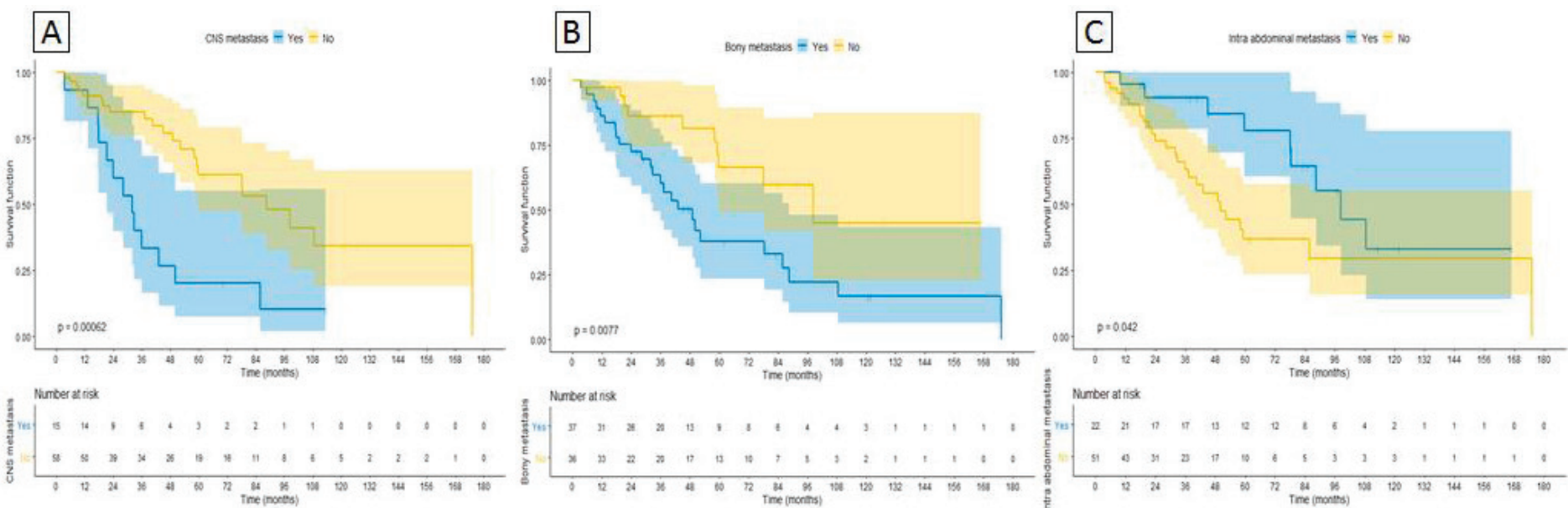

Figure 3. Sites of metastasis correlated with OS. (A): CNS metastasis. (B): Bony metastasis. (C): Intra-abdominal metastasis.

Table 5. Univariable and multivariable analysis.

\begin{tabular}{|c|c|c|c|c|c|c|c|}
\hline \multirow{2}{*}{ Variable } & \multirow{2}{*}{ Category } & \multirow{2}{*}{$N(n=74)$} & \multirow{2}{*}{ Death $(n=35)$} & \multicolumn{2}{|c|}{ Univariate analysis } & \multicolumn{2}{|c|}{ Multivariate analysis } \\
\hline & & & & $\mathrm{HR}(95 \% \mathrm{Cl})$ & $p$ & HR & $p$ \\
\hline \multirow[t]{2}{*}{ CM } & CM & 37 & 16 & $0.246(0.113-0.533)$ & $<0.0001$ & $0.217(0.095-0.497)$ & $<0.0001$ \\
\hline & No-CM & 37 & 19 & & & & \\
\hline \multirow[t]{2}{*}{ Bone metastasis } & Yes & 37 & 25 & $2.638(1.257-5.535)$ & 0.010 & $1.935(0.850-4.405)$ & 0.116 \\
\hline & No & 37 & 10 & Ref & & & \\
\hline \multirow[t]{2}{*}{ CNS metastasis } & Yes & 16 & 13 & $3.176(1.580-6.383)$ & 0.001 & $2.022(0.913-4.474)$ & 0.083 \\
\hline & No & 58 & 22 & Ref & & & \\
\hline \multirow[t]{2}{*}{ Intra-abdominal metastasis } & Yes & 22 & 9 & $0.454(0.208-0.989)$ & 0.040 & $0.620(0.243-1.585)$ & 0.318 \\
\hline & No & 52 & 26 & Ref & & & \\
\hline \multirow[t]{3}{*}{ IMDC } & Good prognosis & 21 & 8 & $0.153(0.044-0.526)$ & 0.003 & $0.298(0.077-1.158)$ & 0.081 \\
\hline & Intermiate prognosis & 49 & 23 & $0.184(0.061-0.556)$ & 0.003 & $0.307(0.093-1.008)$ & 0.052 \\
\hline & Poor prognosis & 4 & 4 & Red & & & \\
\hline
\end{tabular}

\section{Discussion}

In the present study, we found that complete surgical resection of metastasis in clear cell RCC significantly improves OS when compared to systemic treatment alone. This benefit was restricted to IMDC good/intermediate prognosis patients and was not present in the poor prognosis group. We also found that patients undergoing $\mathrm{CM}$ often use less systemic therapy than patients on the no-CM group, suggesting that CM prolongs time-to-treatment-initiation.

Our results are in agreement with prior retrospective studies, supporting survival advantage for patients undergoing metastasectomy [4-9]. However, this is the first study to report results on a homogeneous cohort of patients treated with TT and balanced by IMDC criteria.

Even though no randomised clinical trials have evaluated the role of complete surgical metastasectomy (CM) in mRCC, observational studies-including case series, cohort studies and meta-analysis-have shown important survival benefit in favour of aggressive treatment of 
oligometastatic RCC. These previous reports were mostly conducted in a time when interleukin- 2 and interferon were the standard systemic treatments and few patients had been treated with TT. Alt et al [17] analysed 883 patients with mRCC submitted to multiple complete surgical resections of the metastatic sites. Only 404 (45\%) patients received systemic treatment and $54(13 \%)$ received TT. They demonstrated a significant benefit in OS for pts who had CM analysing the overall cohort (4.8 years versus 1.3 years; $p<0.001$ ). Jakubowski et al [18] evaluated patients submitted to isolated CM and found a 5 year cancer-specific survival (CSS) of 84\%, but they have not reported systemic treatment used or IMDC risk factors of their population, challenging a more elaborated interpretation of results. Zaid et al [19] published a metaanalysis with 2,267 patients and found increased overall mortality with an $\mathrm{HR}$ of $2.37(95 \% \mathrm{Cl} 2.03,2.87 p<0.001)$ for the no-CM group compared with those who underwent CM. Sun et al [20] recently reported a large analysis including 6,994 patients with mRCC, of which $28 \%$ underwent metastasectomy at some point of their disease. They found increase survival for the CM subgroup ( $\mathrm{HR} 0.8395 \% \mathrm{Cl} 0.77-0.90$ $p<0.001$ ). However, both Zaid et al [19] and Sun [20] et al did not specify types of systemic treatment nor site of metastasectomy. They also did not have available IMDC risk factor data and is possible that groups are not well-balanced by these prognostic variables.

It remains uncertain whether this survival benefit previously demonstrated is due to surgical approach itself or to the better performance and prognosis of patients referred to surgery. Therefore, we decided to perform a matched paired analysis, balancing IMDC criteria, age and gender in both groups. This strategy reduced the number of participants, but, on the other hand, balanced the arms, reducing possible selection bias.

CM seems to only benefit patients with good or intermediate prognosis suggesting no survival benefit in poor prognosis patients. These findings need confirmation on a larger cohort, composed by a larger number of poor IMDC risk factor patients, and this has inherent difficulties, as patients with poor prognosis are usually in worse performance-status, not being offered $\mathrm{CM}$.

Furthermore, herein, we discuss in details lines of treatment offered to 74 patients with mRCC, including 37 submitted to curative intent $\mathrm{CM}$, and 37 who did not undergo metastasectomy and were treated with TT alone. Within the CM cohort, 10 patients (27\%) are currently in NED status and have not received any systemic treatment to date. This is important as those patients who would otherwise be exposed to systemic treatments could be spared of potential serious treatment-related adverse events. This finding suggests that surgery can be a definitive treatment option to a very well-selected group of subjects.

The strength of our study lies on the homogeneous cohort of metastatic ccRCC patients, without sarcomatoid or rhabdoid features, and balanced by IMDC, age and gender. Half of the cohort underwent CM and the other half received only TT. The benefit of CM is consistent in univariable and multivariable analysis. This is the first study to allow such a detailed analysis of the population undergoing metastasectomy.

We acknowledge several limitations, including the retrospective and non-randomised nature, and small sample size. Further studies, ideally prospective and randomised, with a larger sample size able to include a reasonable number of IMDC poor-risk patients, are of great importance. The decision to treat oligometastatic patients aggressively versus standard of care systemic therapy is still not answered, especially in poor-risk patients. Our small sample size of poor-risk patients despite not allowing definitive interpretations, suggests a lack of benefit from $\mathrm{CM}$, in line of what was demonstrated by Heng et al [13] for $\mathrm{CN}$.

In summary, we have shown that $\mathrm{CM}$ has a significant impact on survival and remains an important treatment option in mRCC management. Our results suggest that $\mathrm{CM}$ benefit is restricted to good and intermediate IMDC risk patients. In addition, patients who undergo CM are less likely to require systemic treatment, suggesting a role in delaying treatment and avoiding further toxicities.

\section{Conclusion}

Among patients with $\mathrm{mRCC}$ with pairs matched by IMDC, metastasectomy was associated with reduced odds of receiving systemic treatment and with a significant survival benefit. This benefit was significant mainly for favourable and intermediate-risk IMDC but was absent for the poor-risk group. Further studies with larger sample size and, in particular, including poor-risk patients are needed to guide clinical decision-making.

\section{Funding declaration}

No financial support was necessary for this study. 


\section{Conflicts of interest}

None of the authors have any conflicts of interest.

\section{References}

1. Shek D, Tomlinson B, and Brown M, et al (2012) Epidemiologic trends in renal cell carcinoma in the cytokine and post-cytokine eras: a registry analysis of 28,252 patients Clin Genitourin Cancer 10(2) 93-98 https://doi.org/10.1016/j.clgc.2012.01.007 PMID: 22382008

2. Dabestani S, Marconi L, and Hofmann F, et al (2014) Local treatments for metastases of renal cell carcinoma: a systematic review Lancet Oncol 15(12) e549-e561 https://doi.org/10.1016/S1470-2045(14)70235-9 PMID: 25439697

3. Stenman M, Sinclair G, and Paavola P, et al (2018) Overall survival after stereotactic radiotherapy or surgical metastasectomy in oligometastatic renal cell carcinoma patients treated at two Swedish centres 2005-2014 Radiother Oncol 127(3) 501-506 https://doi. org/10.1016/j.radonc.2018.04.028 PMID: 29754859

4. Karam JA, Rini BI, and Varella L, et al (2011) Metastasectomy after targeted therapy in patients with advanced renal cell carcinoma J Urol 185(2) 439-444 https://doi.org/10.1016/j.juro.2010.09.086

5. Kim DY, Karam JA, and Wood CG Role of metastasectomy for metastatic renal cell carcinoma in the era of targeted therapy World J Urol 32(3) 631-642 PMID: 24744223

6. Staehler M (2011) The role of metastasectomy in metastatic renal cell carcinoma Nat Rev Urol 8(4) 180-181 https://doi.org/10.1038/ nrurol.2011.30 PMID: 21475328

7. Bex A, Powles T, and Karam JA (2016) Role of targeted therapy in combination with surgery in renal cell carcinoma Int J Urol 23(1) 5-12 https://doi.org/10.1111/iju.12891

8. Kavolius JP, Mastorakos DP, and Pavlovich C, et al (1998) Resection of metastatic renal cell carcinoma J Clin Oncol Off J Am Soc Clin Oncol 16(6) 2261-2266 https://doi.org/10.1200/JCO.1998.16.6.2261

9. Eggener SE, Yossepowitch O, and Kundu S, et al (2008) Risk score and metastasectomy independently impact prognosis of patients with recurrent renal cell carcinoma J Urol 180(3) 873-878 https://doi.org/10.1016/j.juro.2008.05.006 PMID: 18635225 PMCID: 2570431

10. Ma Y, Yang J, and Qin K, et al (2019) Resection of pancreatic metastatic renal cell carcinoma: experience and long-term survival outcome from a large center in China Int J Clin Oncol https://doi.org/10.1007/s10147-019-01399-w PMCID: 6525119

11. Ljungberg B (2013) The role of metastasectomy in renal cell carcinoma in the era of targeted therapy Curr Urol Rep 14(1) 19-25 https:// doi.org/10.1007/s11934-012-0293-6

12. Verbiest A, Couchy G, and Job S, et al (2018) Molecular subtypes of clear-cell renal cell carcinoma are prognostic for outcome after complete metastasectomy Eur Urol 74(4) 474-480 https://doi.org/10.1016/j.eururo.2018.01.042 PMID: 29463434

13. Heng DYC, Xie W, and Regan MM, et al (2009) Prognostic factors for overall survival in patients with metastatic renal cell carcinoma treated with vascular endothelial growth factor-targeted agents: results from a large, multicenter study J Clin Oncol 27(34) 5794-5799 https://doi.org/10.1200/JCO.2008.21.4809 PMID: 19826129

14. Heng DYC, Wells JC, and Rini BI, et al (2014) Cytoreductive nephrectomy in patients with synchronous metastases from renal cell carcinoma: results from the international metastatic renal cell carcinoma database consortium Eur Urol 66(4) 704-710 https://doi. org/10.1016/j.eururo.2014.05.034 PMID: 24931622

15. Motzer RJ, Tannir NM, and McDermott DF, et al (2018) Nivolumab plus ipilimumab versus sunitinib in advanced renal-cell carcinoma N Engl J Med 378(14) 1277-1290 https://doi.org/10.1056/NEJMoa1712126 PMID: 29562145 PMCID: 5972549 
16. Motzer RJ, Jonasch E, and Agarwal N (2019) NCCN Guidelines Index Table of Contents Discussion Kidney Cancer 61

17. Alt AL, Boorjian SA, and Lohse CM, et al (2011) Survival after complete surgical resection of multiple metastases from renal cell carcinoma Cancer 117(13) 2873-2882 https://doi.org/10.1002/cncr.25836 PMID: 21692048

18. Jakubowski CD, Vertosick EA, and Untch BR, et al (2016) Complete metastasectomy for renal cell carcinoma: comparison of five solid organ sites: complete metastasectomy for RCC J Surg Oncol 114(3):375-379 https://doi.org/10.1002/jso.24327 PMID: 27338155 PMCID: 5104341

19. Zaid HB, Parker WP, and Safdar NS, et al (2017) Outcomes following complete surgical metastasectomy for patients with metastatic renal cell carcinoma: a systematic review and meta-analysis J Urol 197(1) 44-49 https://doi.org/10.1016/j.juro.2016.07.079

20. Sun L, Zhu Y, and Qian Q, et al (2018) Body mass index and prognosis of breast cancer: An analysis by menstruation status when breast cancer diagnosis Medicine (Baltimore) 97(26) e11220 https://doi.org/10.1097/MD.0000000000011220 